Signal \& Image Processing : An International Journal (SIPIJ) Vol.6, No.3, June 2015

\title{
ALEXANDER FRACTIONAL INTEGRAL FILTERING OF WAVELET COEFFICIENTS FOR IMAGE DENOISING
}

\author{
Atul Kumar Verma ${ }^{1}$ and Barjinder Singh Saini ${ }^{2}$ \\ ${ }^{1}$ M.Tech, Department of ECE \\ Dr. B.R Ambedkar National Institute of Technology, Jalandhar, India \\ ${ }^{2}$ Associate Professor, Department of ECE \\ Dr. B.R Ambedkar National Institute of Technology, Jalandhar, India
}

\begin{abstract}
The present paper, proposes an efficient denoising algorithm which works well for images corrupted with Gaussian and speckle noise. The denoising algorithm utilizes the alexander fractional integral filter which works by the construction of fractional masks window computed using alexander polynomial. Prior to the application of the designed filter, the corrupted image is decomposed using symlet wavelet from which only the horizontal, vertical and diagonal components are denoised using the alexander integral filter. Significant increase in the reconstruction quality was noticed when the approach was applied on the wavelet decomposed image rather than applying it directly on the noisy image. Quantitatively the results are evaluated using the peak signal to noise ratio (PSNR) which was 30.8059 on an average for images corrupted with Gaussian noise and 36.52 for images corrupted with speckle noise, which clearly outperforms the existing methods.
\end{abstract}

\section{KEYWORDS}

Image Denoising, Wavelet Transform, Fractional Calculus, Fractional Integral Filtering

\section{INTRODUCTION}

One of the fundamental challenges in image processing and computer vision is image denoising. Noise is a random signal which corrupts an image at the time of image acquisition. Efficient methods for the recovery of original image from there noisy version is extensively explored in literature [1]. There are two types of model for image denoising namely linear and non-linear. The linear model works well reducing the noise present in flat regions of image but is incapable to preserve the texture and edges examples include Gaussian filter and wiener filter etc. The above limitation is removed using the non-linear models which have better edge preserving capability than linear models. The fractional calculus has been applied by numerous researchers in various fields [2], [3] related to image texture enhancement [4], [5] and [6] and image denoising [7], [8], [9] , [10].The results which were corrupted using these operators showed high robustness against different types of noise. Hu et al. [7],[11] implemented a fractional integral 
Signal \& Image Processing : An International Journal (SIPIJ) Vol.6, No.3, June 2015

filter using fractional integral mask windows on eight directions based on Riemann-Liouville definition of fractional calculus. The efficiency of the method is showed by computing the PSNR=27.35 at Gaussian noise with standard deviation $\sigma=25$ for boat image. Guo et al. [12] proposed an image denoising algorithm based on the Grünwald-Letnikov definition of fractional calculus using fractional integral mask windows. Grünwald and Letnikov achieved fine-tuning, by setting a smaller fractional order and controlled the effect of image denoising by iteration. $\mathrm{G}$. Andria [13] proposed a technique for ultrasound medical image denoising using the Linear filtering of 2-D wavelet coefficients. In this technique the image was decomposed into the approximate and details components and then detail components was denoised using Gaussian filter.

Rest of the paper is organized as follows Section 2 describes the background pertaining to concepts of wavelets and the alexander polynomial. Section 3 outlines the proposed method; the experimental results and discussions, including comparison with other existing approaches are given in Section 4. Finally conclusion is presented in the last section.

\section{MATHMATICAL BACKGROUND}

\subsection{Wavelet Foundation}

The word wavelet has been used for decades in digital signal processing [14]. Our focus is on wavelet decomposition which is useful for the applications such as detecting features, image denoising and image compression etc. A wavelet series expansion is defined as a function in terms of the set of orthogonal basic function. For example in Fourier expansion basis consists of sine and cosine function of different frequencies. Many types of functions that are encountered in practice can be sparsely and uniquely represented in terms of the wavelet series. One such example is $L^{2}(\mathrm{R})$ set of all square integrable function on real numbers $R$. It can be shown daubechies, 1992, that it is possible to construct a function $\varphi(\mathrm{x})$ so that any function $f \in \mathrm{L}^{2}(R)$ can be represented by

$$
f(x)=\sum_{k \in Z} c_{0, k} \phi_{0, \mathrm{k}}(x)+\sum_{j<j} \sum_{k \in Z} d_{j, k} \psi_{\mathrm{j}, \mathrm{k}}(x)
$$

where $c_{0, k}=\int_{R} f(x) \phi_{0, \mathrm{k}}(x) \mathrm{dx}, d_{j, k}=\int_{R} f(x) \psi_{\mathrm{j}, \mathrm{k}}(x) d x, \mathrm{j}$ controls the maximum resolution. The function $\psi_{\mathrm{j}, \mathrm{k}}=2^{j} \psi\left(2^{j} x-k\right)$ is obtained from the mother wavelet $\psi(x)$ by dilation and the translation. The function $\phi_{0, \mathrm{k}}(x)$ is obtained from a function $\phi(\mathrm{x})$ known as father wavelet or scaling function by using dilation and translation formula, $\phi_{0, \mathrm{k}}=\phi(\mathrm{x}-\mathrm{k})$. For two dimensions, the scaling function and the wavelets are defined as follows

$\phi_{j, k, l}(\mathrm{x}, \mathrm{y})=\varphi_{j, k}(\mathrm{x}) \varphi_{j, l}(\mathrm{y})=2^{j} \phi\left(2^{j} x-k, 2^{j} y-l\right)$

$\Psi_{j, k, l}(\mathrm{x}, \mathrm{y})=2^{j} \Psi^{s}\left(2^{j} x-k, 2^{j} y-l\right)$.

Here $\mathrm{s}=\mathrm{h} ; \mathrm{v} ; \mathrm{d}$ are all dimensional details characterized as 
$\Psi_{j, k, l}^{h}(x, y)=\varphi_{j, k}(x) \psi_{j, l}(y)$,

$\Psi_{j, k, l}^{v}(x, y)=\varphi_{j, l}(y) \psi_{j, k}(x)$

$\Psi_{j, k, l}^{v}(x, y)=\psi_{j, l}(y) \psi_{j, k}(x)$.

The set $\left\{\phi_{j, k, l}(x, y)\right\} U\left\{\Psi_{j, k, l}^{h}(x, y), \Psi_{j, k, l}^{v}(x, y), \Psi_{j ; k, l}^{v}(x, y) ; \mathrm{j}, \mathrm{k}, 1 \in \mathrm{Z}\right\}$ is an orthonormal basis for function space $\mathrm{L}^{2}\left(R^{2}\right)$. Therefore any function $f \in \mathrm{L}^{2}\left(R^{2}\right)$ can be expressed as

$f(x, y)=\sum_{k, l \in Z} c_{j 0, k, l} \phi_{j 0, \mathrm{k}, \mathrm{l}}(x, y)+\sum_{i} \sum_{j \geq j 0} \sum_{k, l \in Z} d_{j, k, l}^{i} \Psi_{j, k, l}^{i}(x, y)$.

where $c_{j 0, k, l}$ is scaling coefficient and $d_{j, k, l}^{i}$ for $\mathrm{i}=\mathrm{h} ; \mathrm{v} ; \mathrm{d}$ are wavelet coefficients called the subband coefficients.

The wavelets are widely used in image denoising. In [13] G. Andria, proposed the method to denoise the ultrasonic image, in this method firstly they decompose the image using the Symlet 5 wavelet and then applied the Gaussian filter on the detailed components of images and then after reconstruct the image to computed the PSNR, which is better as compared to directly applying the Gaussian filter on the images. Hence it is clear that, with the use of the wavelets in image denoising, that is very much capable to remove the noise as compared to direct one. In our algorithm, the wavelet decomposition of the image is obtained using Symlet 5 wavelet because this function, indeed, are filters with linear phase [15], and therefore the wavelet coefficients are not affected by linear distortion.

\subsection{Alexander Polynomial}

The Alexander polynomial was proposed by J.W. Alexander in 1923 is a knot invariant in which integer coefficients corresponding to each knot type. Until the Jones polynomial was derived in 1984, the Alexander polynomial was the only best known knot polynomial. It is a fundamental tool which explains the pair of curves known as a Zariski pair. A set of two curves $C_{1}$ and $C_{2}$ of equal degree is employed to depict a Zariski pair. If region exist, then $\mathrm{Q}\left(\mathrm{C}_{\mathrm{i}}\right) \subset \mathrm{P}^{2}$ (projective plane) of $C_{i}, i=1,2$ such that $\left(\mathrm{Q}\left(\mathrm{C}_{1}, \mathrm{C}_{1}\right)\right)$ and $\left(\mathrm{Q}\left(\mathrm{C}_{2}, \mathrm{C}_{2}\right)\right)$ are diffeomorphic, while the set of two $\left(\mathrm{P}^{2}, \mathrm{C}_{1}\right)$ and $\left(\mathrm{P}^{2}, \mathrm{C}_{2}\right)$ are not homeomorphic. Our main objective is to construct mask windows using of the Alexander polynomial and its generalized form.

\section{Definition 1}

The Alexander polynomial is formulate as [16]

$$
\Delta(\mathrm{t})=\prod_{\mathrm{m}=1}^{\mathrm{d}-1} \Delta_{\mathrm{m}}(\mathrm{t})^{\mathrm{l}} \mathrm{m}, \mathrm{m}=1, \ldots ., \mathrm{d}-1
$$

Where $\ell_{\mathrm{m}}$ is positive integer and

$$
\Delta_{\mathrm{m}}(t)=\left(\mathrm{t}-\exp \left(\frac{2 \mathrm{~m} \pi \mathrm{i}}{\mathrm{d}}\right)\right)\left(\mathrm{t}-\exp \left(\frac{-2 \mathrm{~m} \pi \mathrm{i}}{\mathrm{d}}\right)\right)
$$


Signal \& Image Processing : An International Journal (SIPIJ) Vol.6, No.3, June 2015

The details of the parameters setting used in the equations can be found in the work by E. ArtalBartolo [16].

\subsection{Fractional Calculus}

The fractional calculus was proposed by Abel over 300 years ago. Afterwards, physical problems as well as potential theory problems are solved using this technique. Now a days many researchers work to use this technique in all areas of sciences [3]. This subsection deals with some definitions regarding fractional calculus.

\section{Definition 2}

The fractional (arbitrary) order integral of the function $\mathrm{s}$ of order $\beta>0$ is defined by

$$
I_{a}^{\beta} S(t)=\int_{a}^{t} \frac{(t-\tau)^{\beta-1}}{\Gamma(\beta)} s(\tau) d \tau
$$

If $\mathrm{a}=0$, then we write $\mathrm{I}_{0}^{\beta} \mathrm{s}(\mathrm{t})=\mathrm{s}(\mathrm{t}) * \gamma(\mathrm{t})$, where $(*)$ denoted the convolution product,

$\gamma(\mathrm{t})=\frac{(\mathrm{t}-\tau)^{\beta-1}}{\Gamma(\beta)}, \mathrm{t}>0$ and $\gamma(\mathrm{t})=0, \mathrm{t} \leq 0 \quad \& \gamma(\mathrm{t}) \rightarrow \delta(\mathrm{t})$ as $\beta \rightarrow 0$ and $\gamma(\mathrm{t}) \rightarrow \delta(\mathrm{t})$ as $\beta \rightarrow 0$

where $\delta(t)$ is the delta function.

In our algorithm, the mask is created using the fractional calculus with utilizing alexander polynomial. After judging the equations for the mask pixels describe in next section we select the two parameters $\beta$ and $t$ by fine tuning on the basis of PSNR.

\section{PROPOSED MeThOD}

\subsection{Procedure for Decomposition}

In this section, according to our studies the wavelet transform is a tool to decompose [17] an image in sub-sampled images, generally consisting of one low-pass filtered approximation, and details corresponding to a high pass filtering in each direction [18] and [19]. In addition, the second level 2-D discrete wavelet decomposition produces seven sub-images A2, H2, V2, D2, $\mathrm{H} 1, \mathrm{~V} 1$ and D1, where A2 is obtained by low pass filtering and twofold decimation along the row and column direction and H1, H2, V1, V2 and D1, D2 shows the horizontal, vertical and diagonal details respectively, for the second level decomposition of a Noisy image. The approximation A2 are the high-scale, low-frequency components of the image and the details H2, V2, D2, H1, V1 and D1 are the low-scale, high-frequency components. Generally the noise is present in high frequency components because noise is the high frequency signal. Our aim is to denoise these components only rather than complete denoise the noisy image. The decomposition of the Noisy image, $I_{n}$ into second-level using symlet-5 mother functions of wavelet families. The size of the mask window should be minimum (3X3) for reducing the computational time, Therefore, the total filtering time for denoising one detail coefficient is $T_{f}=T_{p} X T_{c}=8 n^{2} T_{c}$. Then, overall complexity measure for all detail coefficients images is denoted by $T_{R}, T_{R}=3 \mathrm{X} \mathrm{LX} T_{f}$ where $\mathrm{L}$ is no. of decomposition level and here we use $\mathrm{L}=2$ to achieve desired results. 
Signal \& Image Processing : An International Journal (SIPIJ) Vol.6, No.3, June 2015

\subsection{Procedure for Filter Design}

The procedure of our filter construction uses the definition 2 which explained in section 2

If $\mathrm{a}=0$, we have

$\mathrm{I}^{\beta} \mathrm{t}^{\mu}=\frac{\Gamma(\mu+1)}{\Gamma(\mu+1+\beta)} \mathrm{t}^{\mu+\beta}, \mu>-1 ; \beta>0$

Further, we generalize the Alexander polynomial as explained in definition 1, utilizing the Mittag-Leffler function as

$E_{\beta}(t)=\sum_{m=0}^{\infty} \frac{t^{m}}{\Gamma(\beta m+1)}$

We obtained,

$\Delta^{\beta}(\mathrm{t})=\prod_{\mathrm{m}=1}^{\mathrm{d}-1} \Delta_{\mathrm{m}}^{\beta}(\mathrm{t})^{l_{\mathrm{m}}}, \mathrm{m}=1, \ldots \ldots \ldots \mathrm{d}-1$

Where $l_{\mathrm{m}}$ is the positive integer and

$\Delta_{\mathrm{m}}^{\beta}(\mathrm{t})=\left(\mathrm{t}-\mathrm{E}_{\beta}\left(\frac{2 \mathrm{~m} \pi \mathrm{i}}{\mathrm{d}}\right)\right)\left(\mathrm{t}-\mathrm{E}_{\beta}\left(\frac{-2 \mathrm{~m} \pi \mathrm{i}}{\mathrm{d}}\right)\right.$

By using (3)_we make set of fractional coefficients of Alexander fractional integral sets as

$\tilde{\Delta}_{1}^{\beta}=\tilde{\Delta}_{11}^{\beta}=\frac{2}{\Gamma(3+\beta)} t^{(2+\beta)}-\frac{\sqrt{3}}{\Gamma(2+\beta)} \mathrm{t}^{(1+\beta)}+\frac{t^{\beta}}{\Gamma(1+\beta)}$

$\tilde{\Delta}_{2}^{\beta}=\tilde{\Delta}_{10}^{\beta}=\frac{2}{\Gamma(3+\beta)} t^{(2+\beta)}-\frac{1}{\Gamma(2+\beta)} \mathrm{t}^{(1+\beta)}+\frac{t^{\beta}}{\Gamma(1+\beta)}$

$\tilde{\Delta}_{3}^{\beta}=\tilde{\Delta}_{9}^{\beta}=\frac{2}{\Gamma(3+\beta)} t^{(2+\beta)}+\frac{t^{\beta}}{\Gamma(1+\beta)}$

$\tilde{\Delta}_{4}^{\beta}=\tilde{\Delta}_{8}^{\beta}=\frac{2}{\Gamma(3+\beta)} t^{(2+\beta)}+\frac{1}{\Gamma(2+\beta)} \mathrm{t}^{(1+\beta)}+\frac{t^{\beta}}{\Gamma(1+\beta)}$

$\tilde{\Delta}_{5}^{\beta}=\tilde{\Delta}_{7}^{\beta}=\frac{2}{\Gamma(3+\beta)} t^{(2+\beta)}+\frac{\sqrt{3}}{\Gamma(2+\beta)} \mathrm{t}^{(1+\beta)}+\frac{t^{\beta}}{\Gamma(1+\beta)}$

$\tilde{\Delta}_{1}^{\beta}=\tilde{\Delta}_{11}^{\beta}=\frac{2}{\Gamma(3+\beta)} t^{(2+\beta)}+\frac{2}{\Gamma(2+\beta)} \mathrm{t}^{(1+\beta)}+\frac{t^{\beta}}{\Gamma(1+\beta)}$

In above fractional sets we choose value of $\mathrm{m}$ is from 1 to 11 because the fact that it is a cyclic index. 
Signal \& Image Processing : An International Journal (SIPIJ) Vol.6, No.3, June 2015

For the implementation of mask windows we uses the integral set based on (4) and taking the values of the fractional powers in the range of $0<\beta \leq 0.7$ and $t>0$, after this we move the constructed mask on noisy image by performing convolution on eight directions because the directions of fractional mask windows are invariant to rotation, which are $180^{\circ}, 0^{\circ}, 90^{\circ}, 270^{\circ}$, $135^{\circ}, 315^{\circ}, 45^{\circ}$ and $225^{\circ}$ and these are labelled as $s_{180}(m), s_{0}(m), s_{90}(m), s_{270}(m), \quad s_{135}(m)$, $s_{315}(m), s_{45}(m)$ and $s_{225}(m)$. Each pixels of the details i.e., horizontal details, vertical details and diagonal details are convolved with the mask windows on eight directions. The magnitude for each filter for each individual image $a_{m}(\mathrm{i}, \mathrm{j})$ can be obtained as follows:

$G_{\emptyset}(\mathrm{i}, \mathrm{j})=\sum_{m=1}^{9} a_{m}(\mathrm{i}, \mathrm{j}) * s_{\emptyset}(m)$

where, $\mathrm{m}=1,2, \ldots, 9$ represents the location of pixel inside each mask window and $\emptyset=180^{\circ}, 0^{\circ}$, $90^{\circ}, 270^{\circ}, 135^{\circ}, 315^{\circ}, 45^{\circ}$, and $225^{\circ}$ are represents mask windows on eight directions.

The final new filtered image based on alexander fractional integral filter (AFI) can be obtained by the summation of all eight convolution results of the magnitudes for each filter (5). This process is apply for all the details of wavelet transformed image and then the resultant of AFI filter of all the details are combined with the approximation to get the resultant denoised image.

\section{The Steps of Proposed Method for Image Denoising are as follows:}

Step 1: Resize the original image to $512 \times 512$ pixels.

Step 2: Add artificial noise to the original image (Gaussian and Speckle noise).

Step 3: Decompose the image into sub-bands.

Step 4: Obtained the coefficients for second level decomposition.

Step 5: Denoise each sub-band, except for the low pass residual band using AFI filter.

Step 6: Combined and obtain the denoised image.

Step 7: Calculate the PSNR between the original image and the denoise image.

\section{Design Steps for AFI Filter}

Step 1: Initialize fractional integral windows of $3 \times 3$ sizes.

Step 2: Define the values of the fractional powers of the mask window with the range of $0 \leq \beta<0.7$ and $\mathrm{t}>0$.

Step 3: By setting the optimal value for $\beta=0.52$ shown in Fig.8 and the value of $t=0.54$ can be selected to get the maximum PSNR.

\section{EXPERIMENTAL RESULTS AND EXPLANATION}

\subsection{Database}

The experiments are performed on MATLAB 7.12.0 (R2011a) and windows platform. The proposed algorithm is tested on the standard images taken from [20], [21] include grayscale images, color images and ultrasonic image. The AFI filter is considered to operate using $3 \times 3$ processing mask window.

\subsection{Performance Measure}

The performance of the proposed filtering method was evaluated by computing the PSNR. The PSNR is characterized through the mean squared error (MSE) for two images, namely, I and K, 
Signal \& Image Processing : An International Journal (SIPIJ) Vol.6, No.3, June 2015

where one of the images is considered the Original image (or corrupted) and the other is the denoised image respectively.

$$
\operatorname{MSE}=\frac{1}{M N} \sum_{i=1}^{M} \sum_{j=1}^{N}[\mathrm{I}(i, j)-\mathrm{K}(i, j)]^{2}
$$

$\operatorname{PSNR}=10 \log _{10}\left(\frac{\max (I, K)^{2}}{M S E}\right)$

where, $\mathrm{M}, \mathrm{N}$ is the sizes of the images in the rows and columns. They must have same size to obtain the PSNR.

\subsection{Choice of Fractional Power Parameter}

The fractional power parameter used in our method is $\beta$, from the selected value of $\beta$ we decide the pixels of masks. We analyze the behaviour of PSNR for the values of $\beta$, taken from 0.1 to 0.7 , because of the trade-off between PSNR and $\beta$ shown in Fig.1. The maximum PSNR value was obtained by our proposed method using the optimal values of $\beta$ i.e., 0.52 . In our method of image denoising, smaller value of parameter $\beta$ leads to a small value of the PSNR of the denoised image. While an expansive $\beta$ quality prompts sensational reduction of the PSNR. We apply the filter in detailed component of the corrupted image and approximation component is kept untouched because it consists of the low frequency components discussed earlier in section 2.1.

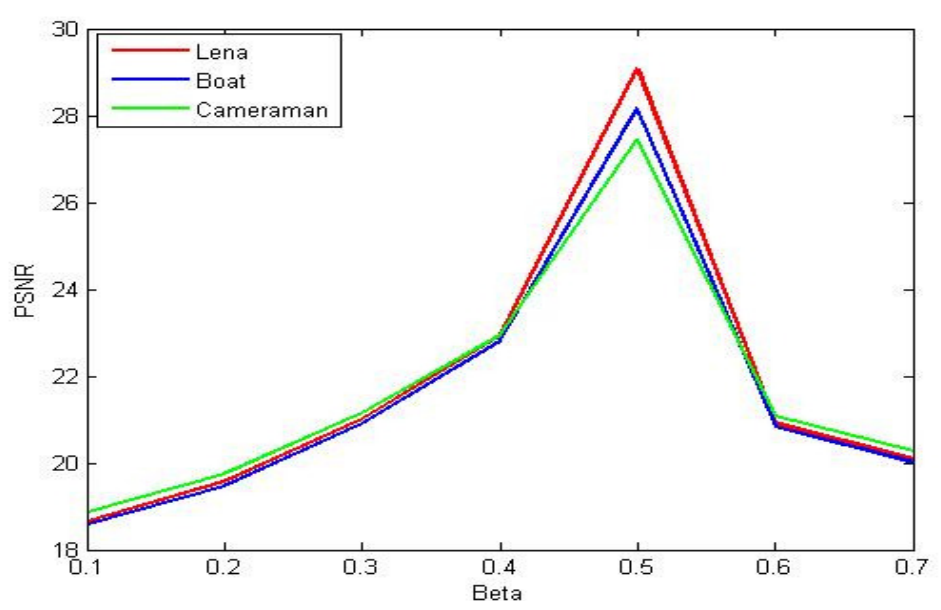

Fig 1: PSNR versus Order plot for grayscale images corrupted by Gaussian noise with standard deviation $\sigma=25$

The better denoising is obtained for $\beta=0.52$ at which selected value of $\mathrm{t}=0.54$ as compared to previous methods.

\subsection{For Visual Perception}

For the human visual perception, we perform the two sets of experiments by adding different noises to the original images which are: 
Signal \& Image Processing : An International Journal (SIPIJ) Vol.6, No.3, June 2015

\subsubsection{Addition of Gaussian Noise}

We perform the experiments to add artificial Gaussian noise with different standard deviations $(15,20$ and 25$)$ to the original standard images. For the standard deviation, $\sigma=15$ we add the Gaussian noise to the Lena and pepper images. The corrupted decomposed detail components of image is passed through the AFI filter and after filtering finally, reconstruct the decomposed image to get the final image. In Fig 2 and Fig 3 we shows the comparison of proposed method with Gaussian filter, AFI and AFD filter visually by passing corrupted image directly to the filter.

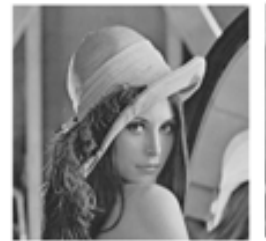

(a)

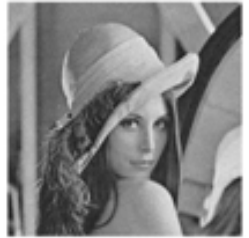

(b)

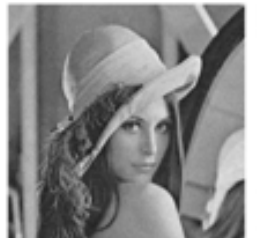

(c)

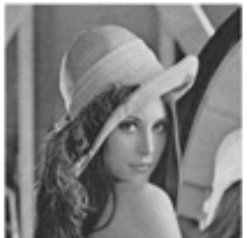

(d)

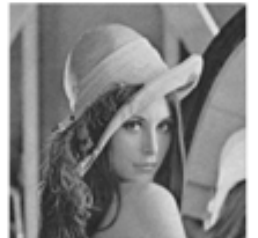

(e)

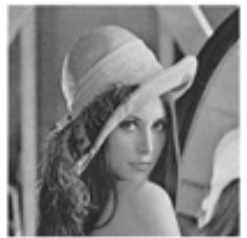

(f)

Fig 2 Results of Grayscale image Lena for visual perception (a) Original Image, (b) Image with Gaussian noise, $\sigma=15$, (c) Gaussian smoothing filter, (d) AFD filter (e) AFI filter (f) Proposed filtering method.

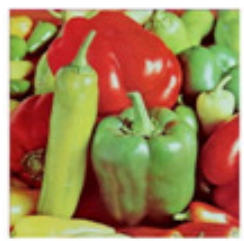

(a)

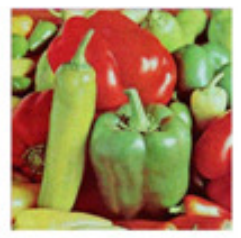

(b)

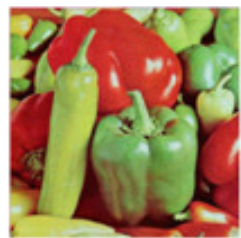

(c)

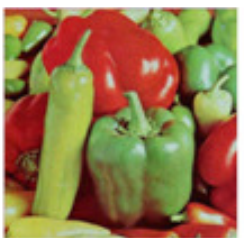

(d)

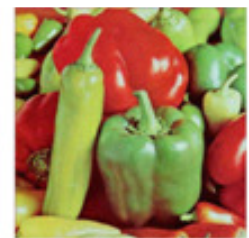

(e)

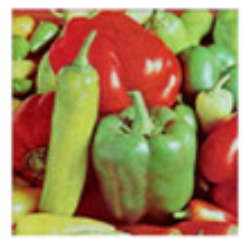

(f)

Fig 3 Results of Color image Peppers for visual perception. (a) Original Image, (b) Image with Gaussian noise, $\sigma=15$. (c) Gaussian smoothing filter, (d) AFD filter, (e) AFI filter, (f) Proposed filtering method.

For the standard deviation, $\sigma=20$ we add the Gaussian noise to the boat and baboon images. The corrupted decomposed detail components of image are passed through AFI filter and then reconstruct the decomposed image to get the final image. In Fig 4 and Fig5 we shows the comparison of proposed method with Gaussian filter, AFI and AFD filter visually by passing corrupted image directly to the filter.

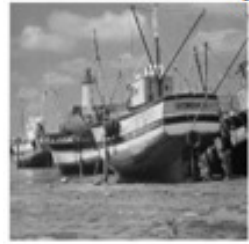

(a)

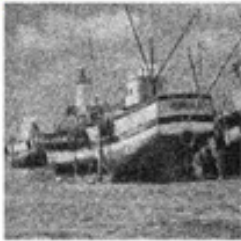

(b)

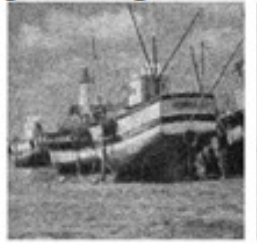

(c)

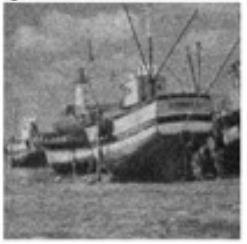

(d)

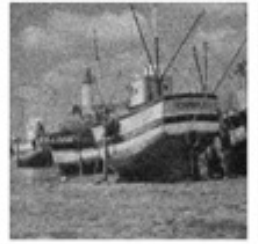

(e)

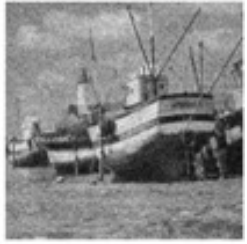

(f)

Fig 4 Results of Grayscale image Boat for visual perception (a) Original Image, (b) Image with Gaussian noise $\sigma=20$. (c) Gaussian smoothing filter, (d) AFD filter, (e) AFI filter, (f) Proposed filtering method. 
Signal \& Image Processing : An International Journal (SIPIJ) Vol.6, No.3, June 2015

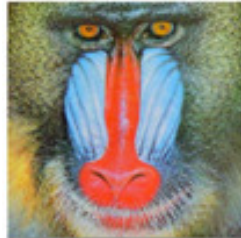

(a)

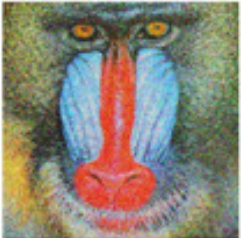

(b)

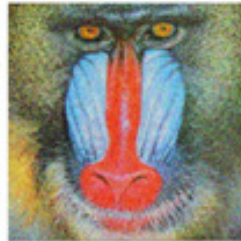

(c)

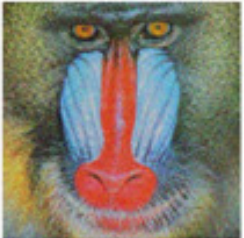

(d)

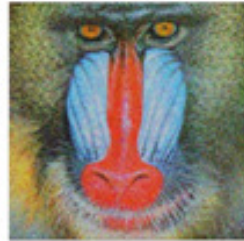

(e)

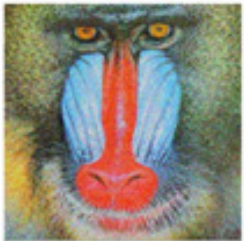

(f)

Fig 5 Results of Color image Baboon for visual perception (a) Original Image, (b) Image with Gaussian noise $\sigma=20$, (c) Gaussian smoothing filter, (d) AFD filter, (e) AFI filter, (f) Proposed filtering method.

For the standard deviation, $\sigma=25$ we add the Gaussian noise to the Cameraman and House images. The corrupted decomposed detail components of image are passed through the AFI filter for denoise and then reconstruct the decomposed image to get the final image. In Fig 6 and Fig 7 we shows the comparison of proposed method with Gaussian filter, AFI and AFD filter visually by passing corrupted image directly to the filter.

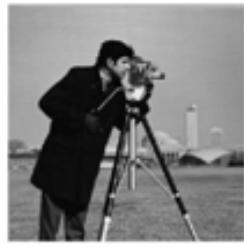

(a)

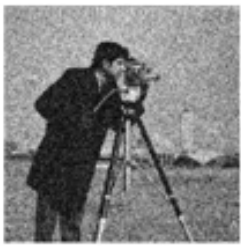

(b)

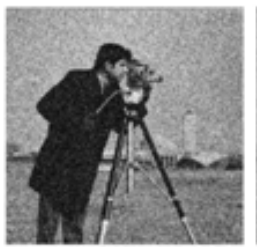

(c)

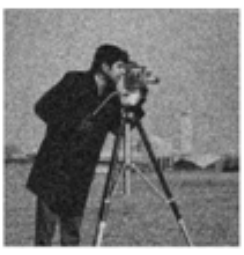

(d)

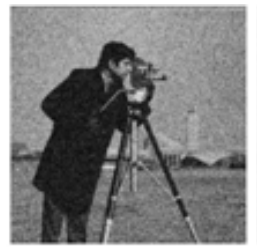

(e)

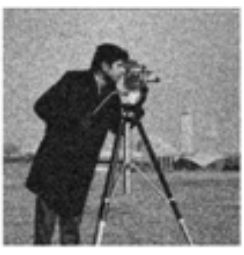

(f)

Fig 6 Results of Grayscale image Cameraman for visual perception (a) Original Image, (b) Image with Gaussian noise $\sigma=25$. (c) Gaussian smoothing filter, (d) AFD filter. (e) AFI filter, (f) Proposed filtering method.

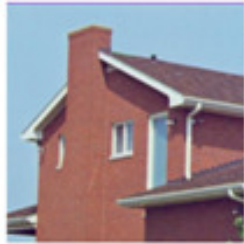

(a)

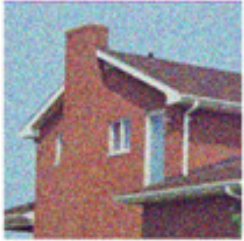

(b)

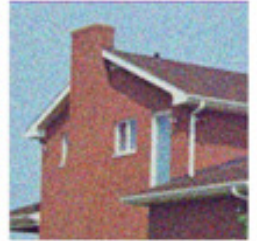

(c)

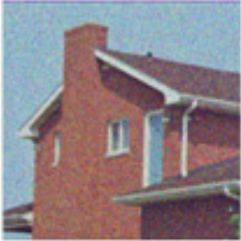

(d)

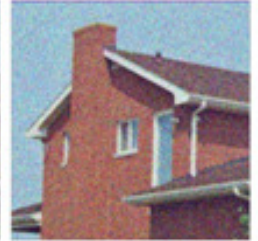

(e)

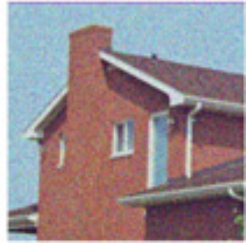

(f)

Fig 7 Results of Color image House for visual perception (a) Original Image, (b) Image with Gaussian noise $\sigma=25$, (c) Gaussian smoothing filter, (d) AFD filter, (e) AFI filter, (f) Proposed filtering method

\subsubsection{Addition of Speckle Noise}

We perform the experiments to add speckle noise with variance $=0.04$ to the ultrasonic images When the corrupted decomposed detail components of image is passed through the AFI filter to denoise and then reconstruct the decomposed image to get the final image. In Fig 8 we shows the comparison of proposed method with Kuan filter, AFI and AFD filter visually by passing corrupted image directly to the filter. 
Signal \& Image Processing : An International Journal (SIPIJ) Vol.6, No.3, June 2015

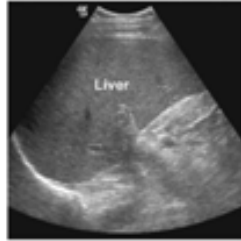

(a)

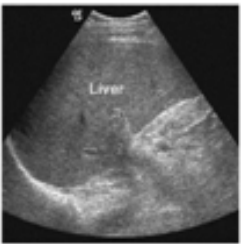

(b)

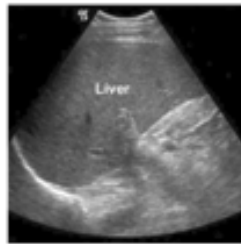

(c)

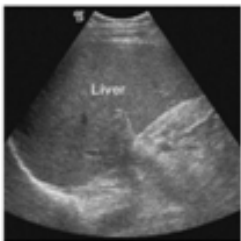

(d)

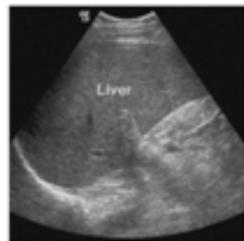

(e)

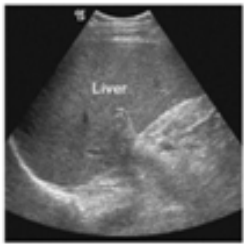

(f)

Fig 8 Results of Ultrasonic image Liver for visual perception (a) Original Image, (b) Image with speckle noise variance $=0.04$, (c) Gaussian smoothing filter, (d) AFD filter, (e) AFI filter, (f) Proposed filtering method.

\subsection{Quantitative Comparison with other Methods}

For the quantitative comparison purpose we measure the PSNR between the Original and denoised images for the standard images corrupted by the gaussian noise. The Table1 tells the PSNR value of our proposed filtering method is higher than the previous method and shows better results than Gaussian, AFD and AFI filters.

\begin{tabular}{|c|c|c|c|c|c|}
\hline \multicolumn{2}{|c|}{ TABLE 1 Comparison of PSNRs obtained by different image denoising methods } \\
\hline \multirow{2}{*}{$\begin{array}{l}\text { Images } \\
(512 X 512)\end{array}$} & $\begin{array}{c}\text { Gaussian } \\
\text { Noise } \sigma\end{array}$ & $\begin{array}{c}\text { Gaussian } \\
\text { Filter } \\
{[20]}\end{array}$ & $\begin{array}{c}\text { AFD } \\
{[20]}\end{array}$ & $\begin{array}{c}\text { AFI } \\
{[20]}\end{array}$ & $\begin{array}{c}\text { Proposed Filtering } \\
\text { Method }\end{array}$ \\
\cline { 3 - 6 } & 15 & 28.23 & 29.06 & 29.53 & 36.10 \\
\hline Lena & 15 & 28.14 & 29.67 & 29.05 & 30.05 \\
\hline Pepper & 20 & 25.73 & 28.66 & 28.97 & 32.72 \\
\hline Boat & 20 & 24.87 & 25.74 & 25.66 & 27.54 \\
\hline Baboon & 25 & 24.24 & 26.47 & 27.01 & 31.35 \\
\hline Cameraman & 25 & 24.03 & 26.61 & 27.00 & 27.08 \\
\hline House & & & & & \\
\hline
\end{tabular}

Table 2 shows the result of PSNR obtained for the ultrasonic image corrupted by the artificial speckle noise is much better as compared to the Kuan, AFD and AFI filters. The reason for higher PSNR achieve, is that when using the concept of wavelet with AFI filter, which only affects the pixel values that are changing sharply (high frequency of image), while no significant changes happen in low frequency of image [17]

\begin{tabular}{|c|c|c|c|c|c|}
\hline \multirow[b]{3}{*}{ Image } & \multirow[b]{3}{*}{$\begin{array}{l}\text { Speckle } \\
\text { Variance }\end{array}$} & \multirow{2}{*}{\multicolumn{4}{|c|}{$\operatorname{PSNR}(\mathrm{dB})$}} \\
\hline & & & & & \\
\hline & & $\begin{array}{c}\text { Kuan } \\
\text { Filter } \\
{[20]} \\
\end{array}$ & $\begin{array}{l}\text { AFD } \\
{[20]}\end{array}$ & $\begin{array}{l}\text { AFI } \\
{[20]}\end{array}$ & $\begin{array}{l}\text { Proposed } \\
\text { Filtering } \\
\text { Method } \\
\end{array}$ \\
\hline Ultrasonic & 0.04 & 32.02 & 33.28 & 33.40 & 36.52 \\
\hline
\end{tabular}

For the Tables 3 we compute the PSNR between the corrupted and denoised image because of the comparison of our proposed method with the Fractional integral method [8] in which PSNR is 
Signal \& Image Processing : An International Journal (SIPIJ) Vol.6, No.3, June 2015

obtained between the corrupted and denoised image. In this table we show the results of Lena and Boat image when these are corrupted by different artificial Gaussian noise standard deviation $(\sigma=15,20$ and 25). It can be seen from the table for boat and Lena image, the values of PSNR for our proposed filtering method are slightly larger than the methods in [8],[22] corrupted by noise standard deviation $\sigma$ values of 15 and 20. The proposed method for the image denoising gives attractive results when the image is highly corrupted by Gaussian noise. The Higher PSNR of our proposed algorithm acts as one of the important parameters in judging its performance.

\begin{tabular}{|c|c|c|c|c|c|}
\hline \multicolumn{6}{|c|}{$\begin{array}{l}\text { TABLE } 3 \text { Comparison of the experimental results for grayscale Boat and Lena } \\
\text { image with other methods. }\end{array}$} \\
\hline \multirow[b]{2}{*}{$\begin{array}{c}\text { Image } \\
(512 \mathrm{X} \\
512)\end{array}$} & \multirow[b]{2}{*}{$\begin{array}{c}\text { Gaussian } \\
\text { Noise } \\
\sigma\end{array}$} & \multicolumn{4}{|c|}{$\operatorname{PSNR}(\mathrm{dB})$} \\
\hline & & $\begin{array}{c}\text { Fractional } \\
\text { Integral } \\
\text { Filter } \\
{[8]}\end{array}$ & $\begin{array}{l}\text { AFD } \\
{[20]}\end{array}$ & $\begin{array}{l}\text { AFI } \\
{[20]}\end{array}$ & $\begin{array}{c}\text { Proposed } \\
\text { Filtering } \\
\text { Method }\end{array}$ \\
\hline \multirow{3}{*}{ Boat } & 15 & 29.20 & 29.97 & 29.54 & 30.12 \\
\hline & 20 & 27.91 & 28.66 & 28.97 & 29.23 \\
\hline & 25 & 26.97 & 27.39 & 27.90 & 28.12 \\
\hline \multirow{3}{*}{ Lena } & 15 & 29.93 & 29.20 & 29.54 & 30.15 \\
\hline & 20 & 28.01 & 28.27 & 28.97 & 30.08 \\
\hline & 25 & 27.35 & 27.28 & 27.90 & 28.02 \\
\hline
\end{tabular}

\section{CONCLUSION}

In this paper, an image denoising algorithm based on wavelet decomposition with fractional integral is proposed. The denoising performance is measured by performing experiment based on visual perception and PSNR values. The experiments shows that the improvements achieved are compatible with the standard Gaussian smoothing, AFI and AFD filters. An additional interesting property of our proposed method is characteristic of the denoised method that can be adjusted easily by changing the numbers of levels of decomposition and two values of fractional powers of proposed mask windows may be changed. In future studies proposed filter method can be modified for texture enhancement of digital image.

\section{REFERENCES}

[1] M.C. Motwani, et al. Survey of image denoising techniques, in: Proceedings of GSPX, Citeseer, 2004.

[2] A.A. Kilbas, H.M. Srivastava, J.J. Trujillo, Theory and applications of fractional differential equations, Mathematics Studies, vol. 204,Elsevier Science Inc., New York, NY, USA, 2006.

[3] I. Podlubny, Fractional differential equations: an introduction to fractional derivatives, fractional differential equations, to methods of their solution and some of their applications, vol. 198, Academic Press, New York, NY, USA, 1999.

[4] H.A. Jalab, R.W. Ibrahim, Texture enhancement based on the Savitzky-Golay fractional differential operator, Math. Probl. Eng. 2013 (2013) 1-8.

[5] H.A. Jalab, R.W. Ibrahim, Texture enhancement for medical images based on fractional differential masks, Discret. Dyn. Nat. Soc. 2013 (2013) (2013) 1-10.

[6] H.A. Jalab, R.W. Ibrahim, Texture feature extraction based on fractional mask convolution with cesáro means for content-based image retrieval, in: Manuel Duarte Ortigueira( ed.), PRICAI 2012: Trends in Artificial Intelligence, Springer-Verlag, Berlin, Heidelberg, 2012,170-179. 
Signal \& Image Processing : An International Journal (SIPIJ) Vol.6, No.3, June 2015

[7] J. Hu, Y. Pu, J. Zhou, A novel image denoising algorithm based on Riemann-Liouville definition, J. Comput. 6 (7) (2011) 1332-1338.

[8] H.A. Jalab, R.W. Ibrahim, Denoising algorithm based on generalized fractional integral operator with two parameters, Discret. Dyn. Nat. Soc. 2012 (2012) 1-14.

[9] S. Das, Functional Fractional Calculus, Springer Verlag, Berlin, Heidelberg, 2011.

[10] E. Cuesta, M. Kirane, S.A. Malik, Image structure preserving denoising using generalized fractional time integrals, Signal Process. 92 (2)(2012) 553-563.

[11] J. Hu, Y. Pu, J. Zhou, Fractional integral denoising algorithm and implementation of fractional integral filter, J. Comput. Inf. Syst. 7 (3) (2011) 729-736.

[12] K. S. Miller and B. Ross, An Introduction to the Fractional Integrals and Derivatives-Theory and Application, John Wiley\& Sons, New York, NY, USA, 1993.

[13] Ricker, Norman(1953) "Wavelet contraction, wavelet expansion,and the control of seismic resolution".Geophysics 18 (4) . doi: 10.1190 / 1.1437927.

[14] Akansu,AliN; Haddad, Richard A.(1992) ,Multiresolution signal Decomposition: transforms , subbands, and wavelets, Boston, MA: Academic Press,ISBN 978-0-12-047141-6.

[15] E. Pinheiro, O. Postolache, P. Girao, Automatic wavelet detrending benefits to the analysis of cardiac signal acquired on moving wheelchair, in: Proc. of EMBC/10, September 2010, pp 602-605.

[16] E. Artal-Bartolo, Sur les couples de Zariski, J. Algebr. Geom. 3 (1994)223-247.

[17] S.G. Mallat, A theory for multiresolution signal decomposition: thewavelet representation, IEEE Transactions on Pattern Analysis and Machine Intelligence 11 (1989) 674-693.

[18] S.G. Mallat, Wavelet for vision, Proceedings of IEEE 84 (1996) 604-614.

[19] http://www.image processing place. com/root files_V3/image_databases.htm

[20] Hamid A. Jalab Rabha W. Ibrahim, "Fractional Alexander polynomials for image denoising", Elsevier journal of signal processing 107(2015)340-354.

[21] http://decsai.ugr.es/ javier/denoise/testimages

[22] G. Andria, F. Attivissimo, G. Cavone, N. Giaquinto, A.M.L. Lanzolla, "Linear filtering of 2-D wavelet coefficients for denoising ultrasound medical images", Elsevier journal of measurement 45 (2012) 1792-1800. 